\title{
Interactive comment on "Employing airborne radiation and cloud microphysics observations to improve cloud representation in ICON at kilometer-scale resolution in the Arctic" by Jan Kretzschmar et al.
}

Jan Kretzschmar et al.

jan.kretzschmar@uni-leipzig.de

Received and published: 18 September 2020

The response to referee comment 1 is attached as a supplement.

Please also note the supplement to this comment:

https://acp.copernicus.org/preprints/acp-2020-641/acp-2020-641-AC1-supplement.pdf 